\title{
Spatial Power Combining Experiment of High Power Microwave
}

\author{
Yang Jinghong ${ }^{1, a^{*}}$, Xu Chunlin $^{1}$, Yang Ming $^{1}$,Qian Meng ${ }^{1}$, Liu Chao $^{1}$, \\ Liao Yuan ${ }^{1}$,Zheng Xin $^{1}$ and Ma Lin ${ }^{1}$ \\ ${ }^{1}$ Nanjing Research Institute of Electronics Technology, Nanjing 210039, China
}

ajwycumt@163.com

\begin{abstract}
Keywords: High power microwave, spatial power combining, microwave source, phased array antenna, klystron.

Abstract. Spatial power combining scheme of employing multiple coherent high power microwave sources together with phased array antennas is presented to improve effective radiation power of High Power Microwave Source (HPMS). HPMS and spatial power combining validation system are developed, and spatial power combining experiment with 2 sets of HPMSs is conducted. HPMS, based on common high power klystrons and making use of inductive-adder ultra-high-voltage all-solid-state modulators, transmits and distributes its power uniformly to each radiation element of phased array antenna via radial line waveguides, and the spatial direction of transmitting beam is controlled by setting phase value of each radiation element. Experimental result shows that power combining efficiency is better than $90 \%$.
\end{abstract}

\section{Introduction}

High Power Microwave (HPM) usually refers to strong pulse electromagnetic radiation with frequency of 1-300GHz and peak power of more than 100MW [1]. High Power Microwave Weapon (HPMW) indicates that it radiates HPMS-generated RF energy via high-gain antenna with microwave energy concentrated in a narrow beam, and illuminates the target with extremely high electric-field strength (each pulse energy ranges from $1 \mathrm{~kJ}$ to $10 \mathrm{~kJ}$ ), so as to cause damage or destroy outcome. The performance of HPMW depends on the output power of HPMS, the antenna gain, the accuracy of antenna direction, and the target-to-source distance [2].

HPMW requires hundreds of GW microwave power to obtain combat capability, subsequently increasing microwave source output power becomes key technique of HPMW system. Several constraints such as the physical mechanism of high power microwave generation and the process structure of device make it difficult to further increase single HPMS output power, so large antenna aperture is needed. Nevertheless, antenna aperture cannot be very large because of space limit and transport. As a result, it can be concluded that effective radiation peak power is limited [3].

Spatial power combining technique of HPM directs multiple antenna radiation units carrying output power from multiple coherent HPMSs to the target and illuminates the target simultaneously, and the microwave energy adds in space, so the total energy illuminated on target equals to the sum of the energy radiated by multiple single HPMSs, and HPMW output power as well as combat effectiveness is significantly boosted [4].

In this paper, HPMS using common high power klystron and phased array antenna are presented. The high power microwave spatial power combining validation system with the peak power up to 100MW level is developed, and power combining experiment is performed.

\section{Spatial Power Combining Validation System}

High power microwave spatial power combining employs 2 sets of 50MW-peak-output-power HPMSs. Each phased array antenna corresponds to 1 set of HPMS. HPMS output power is uniformly distributed to each radiation element of phased array antenna via power distribution network. The spatial direction of transmitting beam is controlled by shifting phase of radiation elements. Principle of the validation system of spatial power combining experiment is depicted in figure 1 . 


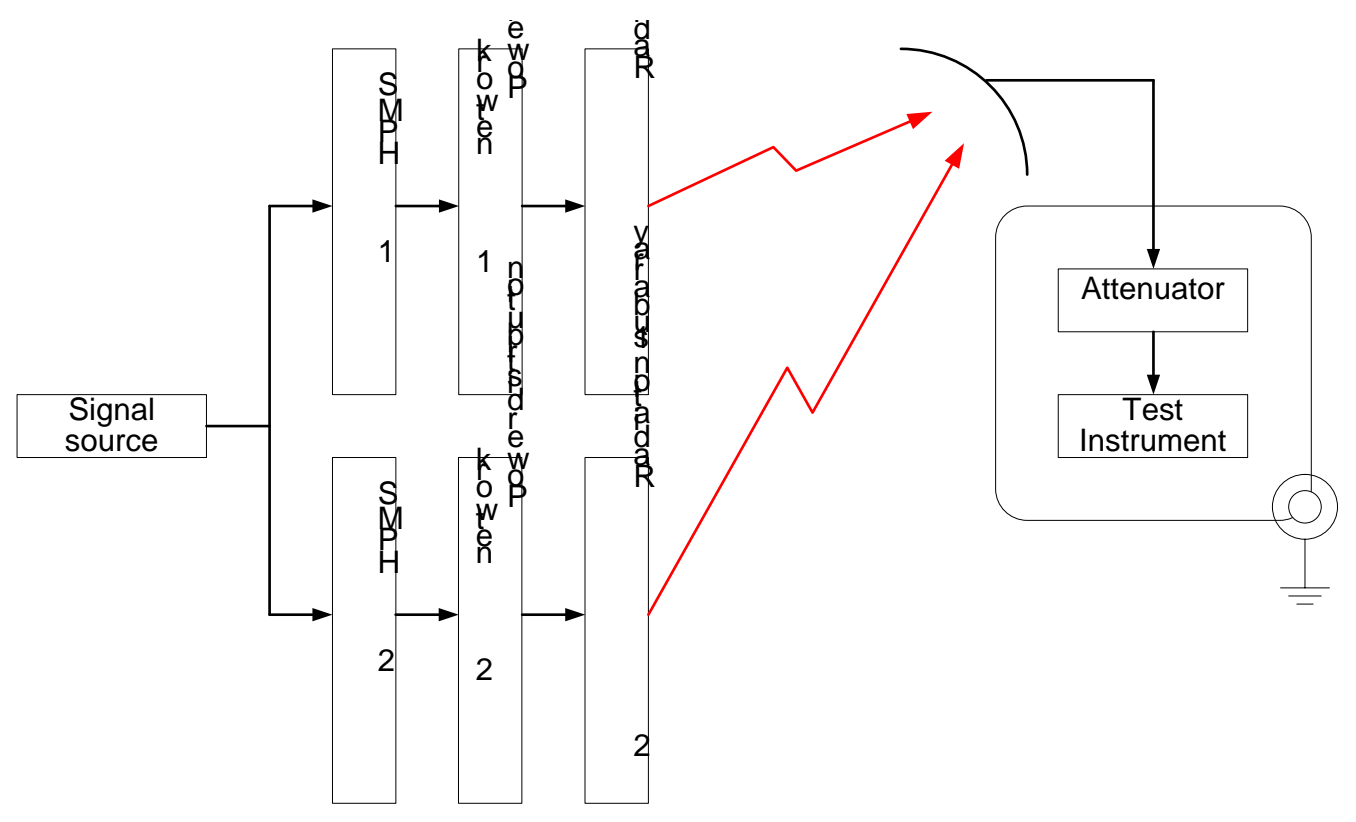

Figure 1. Validation system of spatial power combining experiment.

Key techniques of spatial power combining system include HPMS, power distribution and phase control.

HPMS adopts inductive-adder ultra-high-voltage all-solid-state modulator to drive common high power klystron, and its output peak power exceeds 50MW.

Power distribution network adopts radial waveguide power divider to perform HPMS power transmission and power distribution to each antenna radiation element. Thanks to expansion of radial waveguide size, power endurance capability of radial waveguide itself has improved greatly, being able to bearing hundreds of MW level peak power and with waveguide loss itself of less than $0.5 \mathrm{~dB}$.

Radiation antenna employs motor-driven helical antenna oscillator, which perform phase control taking principle of spatial signal gets $360^{\circ}$ phase shift whenever helical antenna oscillator rotates a circle. It satisfies power endurance and phase adjustment requirement.

\section{HPMS}

The principle of HPMS is illustrated in figure 2. The keys for HPMS are common high power klystron and ultra-high power inductive-adder all-solid-state modulator. Common high power klystron features merits of high power, high gain, high stability and mature technique. Besides, common high power klystron is in favor of the achievement of functions such as radar, jammer, and etc[5].

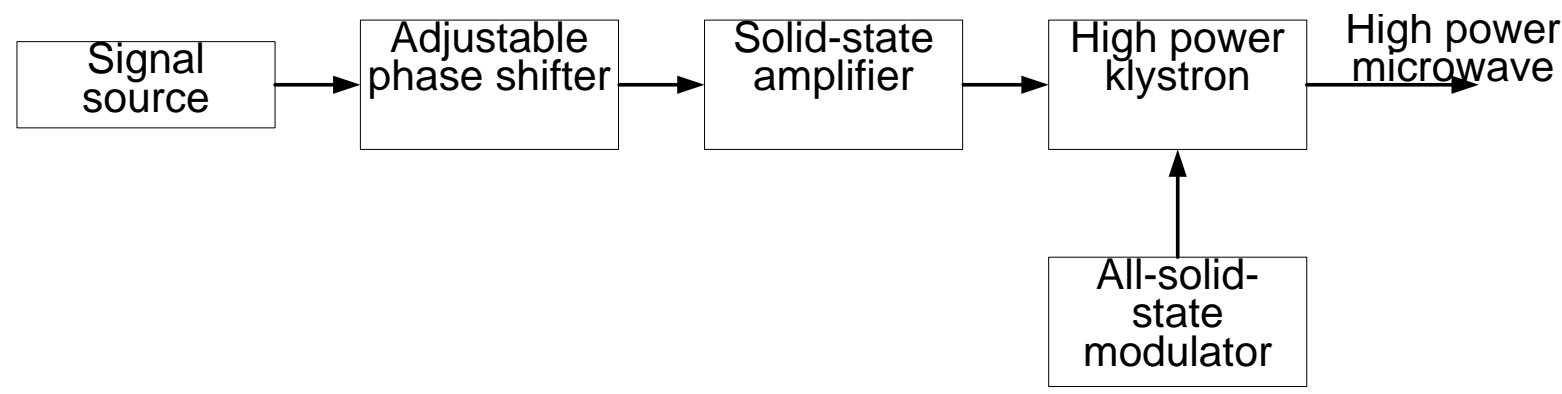

Figure 2. Composition of HPMS.

All-solid-state modulator features merits of small dimension, high efficiency, and convenient maintenance. All the primary hard tube pulse modulators and all the trigger circuit of modulation 
switch in the inductive-adder modulator are identical, working at low potential. Insulation is performed by pulse transformer, so it is unnecessary to consider insulation issues of the modulator. It has much potential to promote modulator reliability because of neither requirement for switch component on/off consistency nor consideration of voltage sharing of switch direct series modulator [6]. Principle of inductive-adder all-solid-state modulator is shown in figure 3.

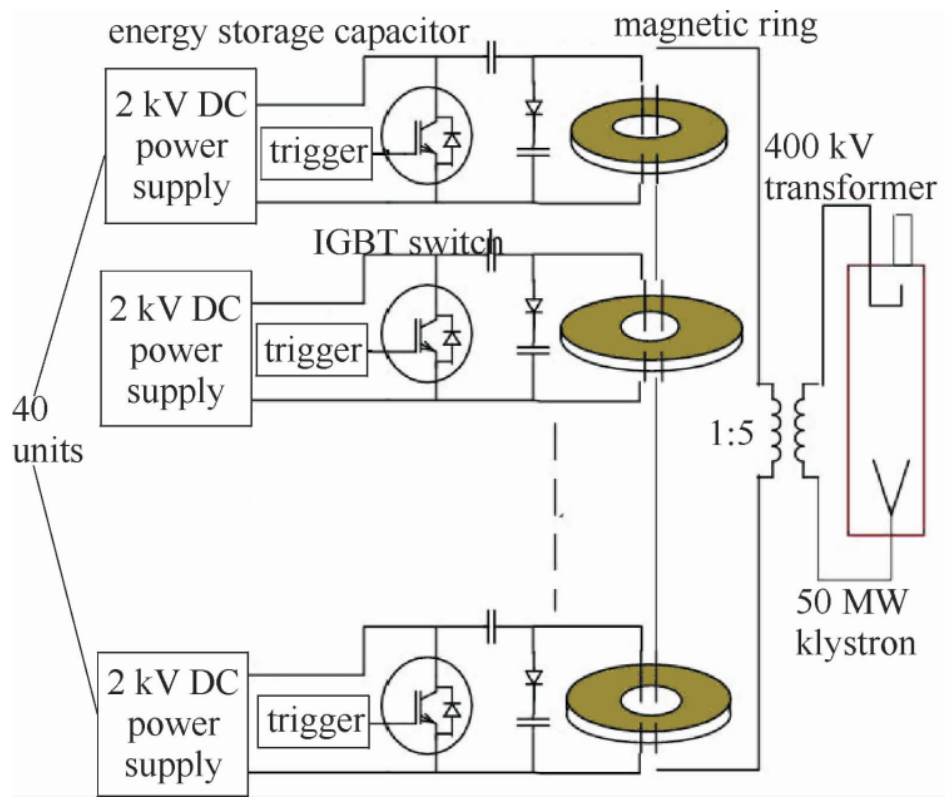

Figure 3. Composition of inductive-adder all-solid-state modulator.

The next step is to develop high power modular all-solid-state modulator which drives 2 ultra-high power common klystrons with several hundreds of MW output power, in this way, a power unit is formed. Furthermore, multiple power units can be combined again to obtain larger-scale HPMS, as shown in figure 4.

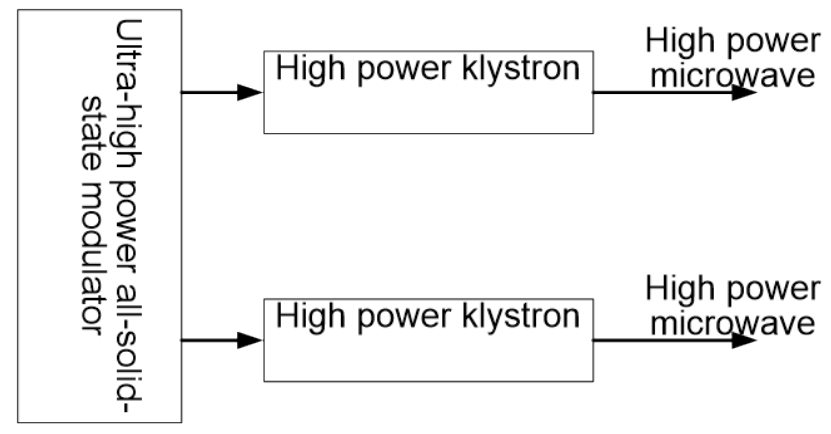

Figure 4. Composition of power unit.

\section{Spatial Power Combining Experiment}

Power combining experiment validation system consists of HPMS, radiation antenna and feeder line, pattern test equipments and so on. Power combining experiment validation system is shown in figure 5 .

2 sets of HPMSs, 2 sets of radiation antennas and feeder line together with its ancillary equipments are installed in a square cabin. The fitting of 2 common high power klystrons should satisfy spatial power combining requirement of 2 sets of antenna arrays. HPMS should possess remote control and operation function. Line distance between cabin and test tower is approximate $600 \mathrm{~m}$. 


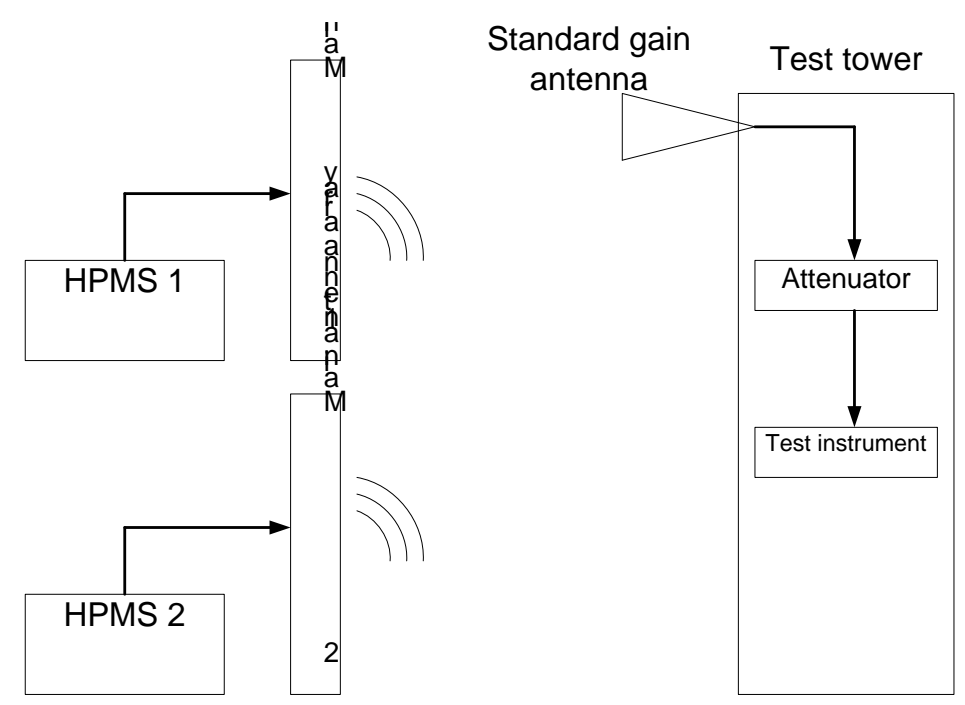

Figure 5. Schematic diagram of power combining experiment.

Test equipments such as receiving antenna (standard gain antenna), connection cable, spectrum analyzer (replacing power meter) are mounted in test tower at the height of $94 \mathrm{~m}$. Test equipments are installed in shielding cage and connected with power attenuators. Radiation antennas scan in a specific angle after HPMSs output power, and receive equipments will record amplitude pattern of the microwave signal received.

Spatial power density of single HPMS as well as that of 2 sets of HPMSs working at the same time can be calculated according to HPMS output power, radiation antenna gain, receiving antenna gain, wavelength, distance between test equipments and radiation antenna, as well as attenuation value caused by attenuators between receiving antenna and test equipments. Spatial power combining efficiency can be calculated when radiation direction of radiation antenna is fixed. With comparing the multiple testing, it shows that power combining efficiency of 2 sets of HPMSs exceeds $90 \%$.

\section{Conclusion}

Spatial power combining of HPMSs is an effective and feasible method to obtain high microwave power. HPM spatial power combining equipments possess flexible combining function, able to radiate power according to required power level. In addition, HPM spatial power combining equipments have excellent mobility and good battlefield survivability.

\section{References}

[1] Liu Yongbo, Fan Xiang, Han Tao. Analysis of the effect mechanism of high power microwave and transmission. Electronic Information Warfare Technology, 2003,18(4):41-45.

[2] He Yuanji, Liu Yanzhi, Li Chuanlu. Study on high power microwave weapons attacking anti-radiation missiles. Aerospace Electronic Warfare,2007,21(4):29-32.

[3] Wang Haiyang. Study on spatial power combining of high power microwave. Chengdu: University of Electronic Science of China,2003:1-6.

[4] Zhang Jiayan, Shu Ting, Yuan Chengwei. Primary study on spatial powers combining of parallel and intersectant beams of high power microwave. High Power Laser And Particle Beams,2007,19(6):915-918.

[5] Yang Jinghong, Qian Meng, Liao Yuan, et al. Spatial power combining microwave source based on single beam klystron. High Power Laser And Particle Beams,2012,24(2):441-444. 
[6] Yang Jinghong, Zheng xin, Qian Meng, et al. Design and Testing of A 160MW High-power Solid-state Modulator [J]. Modern Radar,2011,33(9):72-75. 